\title{
POUR UNE HUMANITÉ À LA RECHERCHE D'ELLE-MÊME : ÉRASME ET LE DÉCLIN DES IDÉOLOGIES
}

Si la prophétie latine, " Nomen Erasmi nunquam peribit » de John Colet, exégète biblique, "Oxford Reformer », doyen de la cathédrale Saint-Paul de Londres et grand ami d'Érasme, n'a pas encore été démentie, près de cinq siècles après qu'elle ait été proférée, ce n'est pas dire que le nom d'Érasme et sa mémoire aient été toujours pieusement honorés, ou simplement traités avec respect tout au long de l'histoire de sa destinée posthume. Plusieurs centenaires de sa naissance ou de sa mort ont pu affecter un calendrier universel ou utopique sans que personne ne se soit avisé de l'événement pour le saluer à sa manière. Il faudra pratiquement attendre le XX" siècle pour que le « prince des humanistes ", la " lumière » ou le " phénix " de l'Allemagne, l' « incomparable génie », alias " apostat », « bouffon qui se jouait des choses les plus saintes », " faune lubrique " " à la paupière hypocrite " (sic) ou " pernicieux coquin " 1, ait droit, de la part des historiens et des chercheurs spécialisés dans l'étude de l'humanisme, de la Renaissance et de la Réforme, non seulement à la célébration de son anniversaire, mais surtout à des jugements fondés sur l'examen approfondi de ses écrits et sur la prise en compte de documents authentiques : ce qui ne signifie pas, tant s'en faut, et Dieu merci, discours uniformes ou interprétations unilatérales ou concordantes de sa pensée.

Lors de la célébration française du demi-millénaire de sa naissance, au mois de juillet 1969, au Centre de la Renaissance de Tours ${ }^{2}$, le regretté Marcel Bataillon - ce prince incontesté des études érasmiennes - tirait la leçon de l'événement, à la suite de la cinquantaine de communications présentces au cours de trois longues semaines. Il disait, dans cette Leçon finale qu'il avait intitulée "Actualité d'Érasme », et qu'il prononçait quelques jours après l'arrivée sur la Lune des deux astronautes américains : "Érasme ne semble avoir conçu aucune admiration enthousiaste pour les hommes de son temps qui faisaient reculer les

1. Parmi les auteurs de ces injures, on peut relever le nom du P. Garasse (jésuite bien connu du XvII* siècle), celui du luthérien Janssen (à la fin du XIX* siècle) ou celui du catholique $\mathrm{L}$. Cristiani (au début du XXo). Ils sont en fait légion.

2. Cf. Marcel Bataillon, "Actualité d'Érasme ", in Colloquia Erasmiana Turonensia, Paris, Vrin/Toronto U. P., 1972, t. II, p. 877-889.

Revue de synthèse : IVe S. N², avril-juin 1987. 
limites du monde connu des Occidentaux anciens et médiévaux... On le voit bien, dans l'Ichtyophagia, jeter un coup d'œil désabusé sur une mappemonde où sont figurées des terres du rivage austral qu'on marque du signe de la croix. C'est pour observer en moraliste : J'ai vu; j'ai appris qu'on avait apporté de là-bas du butin : qu'on y eât introduit le christianisme, je ne l'ai pas entendu dire " ${ }^{3}$. $S^{\prime}$ il avait pu connaître les fêtes commémoratives du $450^{\circ}$ anniversaire de la mort d'Érasme, qui se sont déroulées dans l'Ancien et le Nouveau Monde en 1986 (et qui doivent se prolonger en 1987, par exemple à Lisbonne, au printemps de cette année), nul doute que Marcel Bataillon, pour souligner avec force la signification de la vraie actualité d'Érasme, eût choisi des exemples empruntés à l'actualité des années 1980 , la prolifération des fusées à travers l'espace interplanétaire, les progrès quasi quotidiens de l'électronique et de l'informatique, ou ceux des techniques biologiques qui permettent la naissance d'enfants privés de la présence d'un père ou d'une mère (et parfois des deux) au sens psychoéthique de la paternité ou de la maternité ; et il eût peut-être aussi opposé aux derniers perfectionnements de ces technologies de pointe telle autre réflexion d'Érasme, empruntée, par exemple, à cette épître dédicatoire de son édition de Chrysostome au roi de Portugal Jean III, faisant une fois encore allusion au grand événement de l'époque que fut la liaison maritime établie entre l'Europe et les Amériques : «C'est par leur faute, paraît-il [il s'agit des marins portugais qui ont établi des relations sûres avec l'Inde asiatique], que l'importation étant devenue plus aisće, le prix des denrées n'a pourtant pas baissé, mais au contraire, considérablement augmenté ; et que certaines, comme le sucre, nous arrivent, non seulement plus chères, mais moins bonnes " 4 .

Actualité d'Érasme, certes. Mais que les écologistes ou les socialistes des années 1985 ne s'empressent pas trop d'enrôler Érasme sous leurs bannières. antimonopolistiques ou antitechnologiques, car l'cuvre du Rotterdamois est assez vaste et son esprit assez complexe, ou plutôt nuancé, pour qu'il exalte par ailleurs les progrès accomplis dans tel secteur de la civilisation matérielle, par exemple les manières de table qui permettent aux convives de se tenir plus proprement et de se comporter plus " humainement " dans leurs pratiques alimentaires, ou qu'au contraire cet ami des pauvres ou des humbles ne trouve rien d'autre à dire, en matière d'éducation, quand il est confronté au problème du précepteur à domicile, réservé aux familles riches : «Certes, les enfants pauvres ne pourront pas recourir à ce système éducatif ; mais, comme disait le poète, quand on ne peut pas faire ce que l'on veut, on fait ce que l'on peut! "s

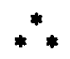

3. Ibid., p. 877 .

4. Ibid., p. 878.

5. Voir à ce sujet, De pueris instituendis, ed. crit. Jean-Claude Margolin, in Opera Omnia Des. Erasmi Roterodami, Amsterdam, North-Holland Publ. Company (cité par la suite comme $A S D), 1971,1-2$, p. 63. 
Ce qui me frappe, avec le léger recul pris par rapport aux grandes manifestations érasmiennes de 1986 auxquelles il m'a été permis d'assister ou sur lesquelles j'ai obtenu le maximum de documents et de renseignements - célébrations et colloques de Paris et de Bale, en avril, de Tours en juillet, de Wolfenbüttel et de Rotterdam en novembre, de l'Institut néerlandais de Paris en décembre, articles de fond des catalogues des expositions de Rotterdam et de Bale, etc. - , c'est le double caractère d'erudition sereine - on trouve toujours du nouveau, malgré les milliers d'études consacrées à Érasme, quand on est un chercheur rigoureux, enthousiaste et patient - et d'actualisation sans agressivité des thèmes ou des débats majeurs d'une cuvre immense, que l'on a enfin abordée et commencé d'approfondir dans toutes ses dimensions, littéraire et philologique, socio-historique, pedagogique, éthique, theologique, voire - avec quelque timidité - psychanalytique. Cette remarque est d'ailleurs valable, bien entendu, pour les travaux érasmiens qui sortent en foule, depuis une quinzaine ou une vingtaine d'années, de tous les ateliers - collectifs ou individuels disséminés dans un très grand nombre de pays d'Europe, d'Amérique, d'Asie - notamment au Japon - ou d'Australie, mais dont quelques points d'élection sont figurés par Toronto (et ses Presses Universitaires), Bruxelles (et ses Presses Universitaires) ou Amsterdam (qui publie, a la North-Holland Publishing Company, depuis 1969 , cette monumentale édition critique, riche aujourd'hui de quinze volumes, qui marquera incontestablement les études érasmiennes des dernières décennies de ce siecle, et sans doute des premières du suivant). Je l'ai souligné personnellement dans deux articles récents, l'un de la revue allemande Daphnis 6, l'autre de la Bibliotheque d'Humanisme et Renaissance ?, montrant que les engagements personnels de certains interprètes d'Érasme - notamment en matière d'exégèse biblique et, plus généralement, d'interprétation religieuse - n'alourdissaient pas, bien au contraire, le climat intellectuel, voire affectif des échanges : qu'on en juge par les comptes rendus critiques des revues ou par les discussions des rencontres internationales. Je pense, avec beaucoup d'autres - et c'est là un fait qui ne saurait laisser indifférents des historiens - que le Concile de Vatican II, la pratique d'un cocumenisme auquel se rallient, bien volontiers, un nombre de plus en plus important de chrétiens - catholiques et protestants, ou encore ces chrétiens orthodoxes, que le pape actuel va visiter chez eux - ainsi qu'une défense authentique et sans concession des droits de l'homme d'un bout à l'autre de la planète, ne sont pas étrangers à ce changement de climat, même au cours de discussions avec des anti-erasmiens (car il en reste !).

Or que constate-t-on dans un nombre important de travaux érasmiens qui ont marqué ces dernières années, ou dans les échanges perçus à travers les récents colloques ? L'actualité érasmienne le plus souvent évoquée, analysée, fouillée et discuté, est celle qui porte sur les problèmes d'éducation, sur ceux de la guerre et de la paix, sur le rôle des femmes dans une société civile encore si fortement

6. "Les travaux érasmiens a travers le monde ", Daphnis (Amsterdam), Bd 13, Heft 3, 1984.

7. "Quinze anntes de travaux érasmiens (1970-1985) ", BHR, XLVIII-3, 1986, p. 585-619. 
marquée par la prépondérance masculine. Et quand on aborde le vaste domaine si controversé de la théologie et de la religion, ce qui retient surtout l'attention, c'est l'attitude d'Érasme - tellement différente de celle de la plupart de ses contemporains, même parmi les érudits - à l'égard des hérétiques (ou prétendus tels), des dissidents (comme nous disons aujourd'hui) ou des " déviants ", de ceux - certains Anciens de prédilection, comme Socrate et Cicéron, Platon et Virgile - qui, n'ayant pu connaître le christianisme en raison de leurs dates d'existence, sont très œecuméniquement enrôlés dans la cohorte des pré-Chrétiens. Une journée tout entière du colloque rotterdamois de novembre 1986 était consacrée à ces problèmes de l'éducation libérale à la manière d'Érasme. Journcé symboliquement passée dans le plus ancien établissement scolaire de Rotterdam, le « Gymnasium Erasmianum », où il fut montré et démontré que si le contenu de l'enseignement primaire, secondaire et supérieur a bien changé depuis l'époque du De ratione studii ${ }^{8}$ ou du De pueris instituendis ${ }^{9}$, en particulier dans la place réservée (ou non) au latin et à la lecture expliquée des auteurs classiques, l'esprit de cet enseignement n'est pas foncièrement différent : souci d'individualiser au maximum l'enseignement, de rapprocher le mâttre de l'élève ou de l'étudiant, de concrétiser le plus possible les matière enseignées, y compris la philosophie dont l'architecture des concepts ne se prête pourtant pas généralement à pareil traitement ; souci - 6 combien actuel ! - des débouchés professionnels, pour lesquels, au temps d'Érasme, la pratique courante du latin, dans la parole et l'écriture, était une condition sine qua non, pour peu qu'on eût de l'ambition et les moyens de la satisfaire : l'équivalent pratique de l'anglais, pour les jeunes gens de cette fin du XX' siècle.

Les problèmes politiques, et plus particulièrement ceux qui ont trait aux relations internationales et aux conflits armés entre nations, n'ont pas manqué, tant à Rotterdam qu'à Bäle, et incidemment à Paris et à Tours, d'interpeller (comme on dit) les auteurs de communications et l'ensemble des participants. C'est même sous le signe de la paix et de la tolérance que fut placée la magnifique exposition de Bale du Muste historique (26 avril-7 septembre 1986), qui donna lieu à la publication d'un non moins beau catalogue, intitulé : Erasmus von Rotterdam, Vorkämpfer für Frieden und Toleranz. Les organisateurs de cette commémoration bâloise avaient pris soin de convier à la fête non seulement les érudits et spécialistes qui avaient du nouveau à apporter, mais le bon peuple, épris de paix et de justice, angoissé devant la prolifération des armes et des engins de destruction accumulés dans ce que l'on appelle parfois les deux camps. Sans doute les esprits libéraux et généreux de notre époque sont-ils plus exigeants, en matière de tolérance et de respect des droits de l'homme que les contemporains d'Érasme, et Érasme lui-même, qui ne versait pas de larmes sur les massacres des anabaptistes de Münster en 1534, et qui considérait que Louis de Berquin, le traducteur français de plusieurs de ses écrits religieux, de la Complainte de la Paix et de la Déclamation des Louenges du mariage, avait bien cherché sa cruelle

8. Strasbourg, M. Schürer, aout 1514, cf. $A S D, 1-2$, p. 79 sqq.

9. Băle, H. Froben, 1529 , cf. $A S D, 1-2$, p. 1 sqq. 
exécution en place de Grève ${ }^{10}$. Sans doute nous est-il également difficile d'avaliser certains propos violemment antisémites que nous trouvons dans la correspondance d'Érasme (comme la « lèpre juive » dont seraient corrompues l'Italie et l'Espagne - allusion plus ou moins discrète aux marranes -, ou les " Juifs pestiférés " d'une lettre à Wolfgang Capiton, théologien d'Ingolstadt), ou même dans son écrit pacifiste de la Complainte de la Paix. Mais il faut, comme on dit, tenir compte de l'esprit du temps, et ne pas oublier que le prétendu « Édit de tolérance » de 1598, connu sous le nom d'Édit de Nantes, ne considérait absolument pas les protestants sur un pied d'égalité avec les catholiques, et qu'en particulier l'Église romaine continuait à considérer la religion réformée comme une hérésie : c'était seulement - mais c'est déjà beaucoup - une mesure de pacification ", faisant de la tolérance une simple acceptation, et non (comme on le veut aujourd'hui) une valeur hautement positive. Décalage des siècles, glissement des problématiques, changement des horizons axiologiques : ces différences inévitables qui séparent le temps d'Érasme du nôtre n'empéchent pourtant pas le recours de nos contemporains à celui qui se proclamait un jour " citoyen du monde". Sans doute le monde peut-il etre pris dans le sens purement ethno-géographique et « mondain » de la terre sur laquelle nous vivons, la terre habitce, l'oikouménè - ou dans un sens universel et eschatologique où le concept engloberait non seulement le ciel et les étoiles, c'est-à-dire l'univers créé par Dieu, mais aussi un espace spirituel et non mesurable, celui sur lequel débouche la méditation érasmienne de la vie et de la mort. Toujours est-il que cette proclamation, qui n'a rien de contingent ou de conjonctural, même si c'est dans une lettre déterminée à Zwingli 12 qu'elle a jailli sous la plume de l'humaniste hollandais - exprime un certain scepticisme, en tout cas un certain détachement à l'égard des particularismes nationaux ou régionaux trop agressifs, à l'égard des frontières politiques, constamment remises en question et déplacées à la suite de différends entre les princes qui nous gouvernent, et d'affreux malheurs supportés par leurs peuples. Comment certaines pages d'Érasme et certaines de ses convictions sur la criminelle inanité des guerres intraeuropéennes (et même des croisades contre les Turcs, trop souvent justifiées au moyen de prétextes hypocrites ${ }^{13}$ ) pourraient-elles laisser indifférents, dans les décennies que nous vivons, les artisans de la construction de l'Europe - Europe politique, Europe économique, Europe de la culture ou de la recherche technique et scientifique - et tous ceux qui y participent, à un degré ou à un autre, du seul fait qu'ils sont européens ? Ce n'est certes pas sans de bonnes raisons érasmiennes et sans cette " pensée de derrière " dont parlait Pascal, que les organisateurs du colloque « Érasme » de Wolfenbüttel avaient choisi en 1986 comme thème commun de réflexions et de discussions : Érasme et l'Europe.

10. Voir la lettre d'Érasme a Charles Utenhove du ler juillet 1529, qui développe sur le thème de la prudence quelque peu timorée une élégie funcbre qui pourrait se résumer en : « Je le lui avais bien dit... " Berquin avait été exécuté le 17 avril 1529.

11. Voir à ce sujet, Mario TuRCHetT1, Concordia o Toleranza, Genève, Droz, 1984.

12. Lettre adressete de Bâle en sept. 1522, in Percy S. Allen et al., Opus Epistolarum Erasmi Roterodami, 12 vols, Oxford, 1904-1956, t. V, ep. 1314.

13. Voir sa Consultatio de Bello Turcis Inferendo, Bale, 1530. 
J'ai déjà fait allusion au Concile de Vatican II, à l'esprit qui l'animait, et aux conséquences qui en ont découlé - et qui continuent de se produire - pour les relations, non seulement entre les diverses communautés et les diverses confessions chrétiennes, mais entre les diverses religions monothéistes, et, par-delà les facteurs de différenciation d'ordre religieux, entre les diverses communautés humaines. On se plaît à imaginer l'attitude ou la réaction d'un Érasme, brusquement transporté dans notre siècle et écoutant les proclamations d'un Jean XXIII ou d'un Jean-Paul II : on aimerait parier qu'il ne se trouverait pas dans le camp des chrétiens " intégristes » qui voudraient figer l'Église à un moment déterminé de son histoire, et dont certains ont pu se montrer, dans telles circonstances, plus catholiques - si l'on peut dire - que le pape. Les grands travaux érasmiens, centrés sur des problématiques religieuses et théologiques (et qui confrontent, par exemple, Érasme et saint Augustin, Érasme et Origène, Érasme et saint Jérôme, Érasme et Luther ; ou qui analysent de près les méthodes d'exégèse biblique du Rotterdamois, la texture et l'esprit de ses Annotations, la dimension mystique de l'Éloge de la folie ou du Banquet religieux), sont, consciemment ou inconsciemment, inspirés de cet "esprit du temps" auquel le Concile de Vatican II a donné une coloration particulière. À une époque où un historien luthérien allemand consacre deux importants volumes à la théologie d'Érasme ${ }^{14}$, marqués par une grande compréhension et une évidente sympathie, et où un jésuite belge écrit un important ouvrage sur le libre et le serf-arbitre 15 , où il confronte sans cesse les positions des deux adversaires, et dớnt il serait difficile de déceler le penchant profond, il ne paraît plus possible d'imaginer, sauf chez quelque interprète, aveugle ou sourd aux problemes humains que pose notre temps, des jugements tels que ceux que l'on pouvait lire sous la plume d'historiens reconnus du XIXe siècle ou même de la première moitié du XX'. Ainsi du protestant E. Haag, qui ne voyait dans ses critiques des abus de l'Église que des plaisanteries ne tirant pas à conséquence; ou du luthérien Janssen qui se déchaîne contre lui avec une rare violence verbale; ou du catholique $L$. Cristiani, qui l'accuse d'hypocrisie et qui lui reproche également ses " passages lascifs", sa " déplaisante mendicité " ou encore sa " vie nomade "; ou du rationaliste (au sens du rationalisme agnostique ou athee) et sceptique J.-B. Pineau qui reste aveugle a la dimension métaphysique et théologique de son héros, et qui fait de sa religion une religion purement morale et intellectuelle 16 . De tels jugements, si manifestement faux ou outrés pour peu que l'on examine les faits et les textes sans préjugés, ont acquis de nos jours un intérêt psychologique ou historique - au sens de l'" esprit du temps " qui armait la plume de ces historiens -, mais ils ont perdu toute valeur philosophique ou épistémologique.

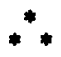

14. Ernst W. KoHLs, Die Theologie des Erasmus, Båle, Reinhardt, 1966.

15. Georges Chantraine, Érasme et Luther : libre et serf-arbitre, Paris/Namur, P. Lethielleux/P.U.N., 1981.

16. Jean-Baptiste PInEAU, Érasme, sa pensée religieuse, Paris, 1924. 
Relisons donc Érasme, en cette fin du XXe siècle, si lourde de menaces de toute sorte et d'angoisse pour une humanité qui cherche encore des règles de vie en commun, et qui commence à se rendre compte que les progrès matériels, techniques et scientifiques risquent de se retourner contre elle et même de la conduire, au sens précis du terme, à sa propre perte, à sa ruine définitive, s'ils ne sont pas maîtrisés par cette conscience - individuelle ou collective - pour laquelle Bergson réclamait déjà un « supplément d'âme ". Sans partager nécessairement son scepticisme usuel à l'égard des grandes découvertes, des progrès de la dynamique ou du déchiffrement des écritures « secrètes », ni celui de Montaigne à l'égard des vérités scientifiques en général (telle vérité, qui a cours à un moment donné, ne l'aura plus demain, telle découverte future recouvre et annihile la découverte d'hier, etc.), nous pouvons encore apprécier la valeur de ce portrait du sage qu'il nous a tracé un jour, dans son colloque des Vieillards ${ }^{17}$, et même l'appliquer à des hommes que n'a pas encore atteints la marque de la vieillesse :

« Je me suis appliqué autant que possible à respecter la formule des Grecs : rester à l'ecart des affaires. Je les ai evitées en général, mais surtout quand elles ne pouvaient se faire qu'en nuisant à autrui. Lorsque j'ai un service à rendre à un ami, je le fais sans me créer du même coup un ennemi. S'il me survient quelque démelé, j'aplanis les difficultés en me justifiant, je les étouffe par quelque service, ou je les laisse assoupir en dissimulant. Je m'abstiens de tout procès ; s'il s'en présente un, j'aime mieux sacrifier l'objet du litige que l'amitié... Je ne fais mauvais visage à personne, je souris à tous, je salue et resalue poliment ; je ne contrarie personne; je ne blâme la manière de vivre ou la conduite de quiconque, je laisse a chacun le loisir de trouver beau ce qu'il possede. Je ne confie à personne ce que je veux tenir secret, mais je ne cherche pas a deviner les secrets des autres; si par hasard j'en apprends un, je me garde de le répéter. Pour les absents, je n'en dis rien, ou bien j'en parle en termes amicaux et sympathiques $\$ "$ ".

Ce portrait de lui-mème, tracé par le vieillard Glycion - l'un de ces quatre vieillards qu'il a plu à la fantaisie d'Érasme de faire voyager dans un coche en 1524, afin qu'ils puissent évoquer tranquillement leurs souvenirs - n'est peut-être pas identifiable à celui du presque sexagénaire humaniste. Et il n'est pas certain que ce repli sur soi et cette réduction du comportement humain à des sentiments élémentaires, même illuminés par l'amitié, constituent un modèle universel susceptible d'entraîner l'enthousiasme de la jeunesse. J'y verrais pourtant, surtout si on le relie à beaucoup d'autres textes d'Érasme - comme celui de la Moria où sont combattues avec une ironie sarcastique, les querelles inexpiables des multiples sectes ou écoles philosophiques ou theologiques - la reconnaissance d'une idée fondamentale : aucune doctrine, aucune religion, aucune idéologie, ne saurait exiger, si attaché qu'on puisse lui être, un déchaînement de violence contre celui qui pense différemment, car derrière l' « ennemi " ou plutôt l'adversaire, il y a l'homme, cré à l'image et à la ressemblance de Dieu.

17. Gerontologia sive Ochèma (ou Senile Convivium), éd. crit. Lbon-E. Halkin et al., in $A S D, 1972, \mathrm{I}-3$, p. 375 sqq.

18. Ibid., p. 378. 
Pour rester sur le terrain spirituel et user du vocabulaire d'Érasme et de ses contemporains, on pourra dire, avec Castellion, que l'hérésie doit être poursuivie, mais que les hérétiques doivent être respectés ou épargnés en tant qu'hommes 19. Transposé en langage moderne et laique, ce principe pourrait s'énoncer : « Combattez par la parole, l'écrit, l'action, ceux dont vous n'approuvez pas les opinions ; mais respectez leurs convictions si elles sont sincères, et surtout respectez leur personne. " Cela s'appelle la démocratie ou la cohésion d'une communauté nationale ou politique fondée sur le pluralisme des opinions ; la démocratie, qui exige, comme on le sait depuis Platon et surtout depuis Montesquieu, un maximum de vertus parmi lesquelles la croyance en la dignité de l'homme et la lutte pour le respect de ses droits. Cette pratique exige l'abolition de tout fanatisme, de toutes les formes d'intolérance, et de la croyance en une idéologie - son idéologie - identifiée à la Vérité. En cette fin du $\mathrm{XX}$ e siècle, qui nous fait assister dans de très larges secteurs de l'opinion à un déclin des idéologies totalitaires - qu'elles soient religieuses, philosophiques, politiques, economiques ou culturelles - et à un rejet des fanatismes subsistants, l'antidogmatisme d'Érasme - qui n'est pas le manque d'assurance ou de certitude métaphysique ou religieuse - convient bien à des esprits très variés : c'est sans doute là l'une des raisons de son succès, qui commence à déborder, sous l'influence des mass-media, la sphère des intellectuels ou des spécialistes. En cette fin de siècle, où l'humanité ne recherche plus de guide éclairé, dépositaire et promoteur d'une vérité unique et totalitaire, il peut être réconfortant de se tourner vers celui que Rabelais appelait, dans une lettre fameuse ${ }^{20}$, 1 ' $"$ invincible champion de la vérité ", celui qui refusait, par souci d'une vérité totale, englobant toute l'humanité - passée, présente et future - de se battre pour des opinions - individuelles et changeantes -, comme Montaigne, un demi-siècle plus tard, quand il déclarait : « Je me contredis bien à l'aventure, mais la vérité, comme disait Demades, je ne la contredis point. "

Jean-Claude MARGOLIN, Universite de Tours, Centre d'études supérieures de la Renaissance.

19. De Haereticis an sint persequendi..., Bâle, 1554 (sous le pseudonyme de Martinus Bellius).

20. Cf. P. S. Allen et al., op. cit. supra n. 12, t. X, ep. 2743 (Lyon, 30 nov. 1532). 